\title{
PREDICTION OF THERMAL STRATIFICATION IN A U-BENT PIPE: A URANS VALIDATION
}

\author{
M. PELLEGRINI ${ }^{1 *}$, H. ENDO ${ }^{2}$, and H. NINOKATA ${ }^{1}$ \\ ${ }^{1}$ Department of Nuclear Engineering, Tokyo Institute of Technology \\ Meguro-ku, Ookayama 2-12-2, N1-5 458, Tokyo, Japan \\ ${ }^{2}$ Japan Nuclear Energy and Safety Organization (JNES) \\ *Corresponding author. E-mail : pellegrini.m.aa@m.titech.ac.jp
}

Received December 30, 2010

Accepted for Publication June 09, 2011

In the present study, CFD is employed to investigate phenomena occurring during a process of thermal stratification in U-bent pipes at transitional Reynolds number. URANS evaluation had been chosen for its low computational costs during transient analysis and for the evaluation of modeling performance in these conditions. Application of CFD at transitional Reynolds number and buoyancy driven flows indeed contains deeper uncertainties in relation to the range of applicability for hydrodynamic and thermal models. The methodology applied in the work points out, through validations with the basic problems constituting the complex stratified phenomenon, the applicability of the current turbulence modeling. Accurate predictions have been found in relation to transitional Reynolds number in bent pipes and region of stability induced by the gravitational field. On the other hand the defects introduced in the unstable region of the U bent pipe, are discussed in relation to the adopted modeling.

KEYWORDS : Thermal Stratification, U bend, CFD Modeling, URANS

\section{INTRODUCTION}

This work investigates the thermal stratification phenomena which occurs in horizontal piping with bends during low flow conditions and after the onset of a thermal gradient at the inlet.

Thermal stratification in nuclear power plants was first recognized from the unexpected movements of the piping system in water reactors during shut-down or start-up of plants [1]. This phenomenon was discovered to be responsible for the creation of thermal stresses and cracking within plants, decreasing their lifetime and safety.

Stratified flows introduce a high load of uncertainty in the physical understanding of their behavior due to difficulties in evaluating them during plant operation or through scaled experiments. In this direction therefore, new experiments were performed at the HTR-facility project [2], [3] employing water as the working fluid. A wide set of experiments was performed for possible transients occurring in LWRs and BWRs, showing the extreme stability of stratification under specific circumstances and pointing out that the layer separating the hot and cold fluid zones is extremely thin compared to the pipe dimension.

The conditions leading to stratification are similar to those occurring in sodium fast reactors (SFRs) in conditions of protected loss of flow; therefore, the same problem was posed in relation to their safety characteristics. Thermal stratification in water and sodium was studied computationally (direct numerical simulation) in [4] for channel geometry and laminar flow. This work points out that, in cases where the temperature plays an important role, the different thermal conductivity between the fluids makes the behavior of the thermal field in fact different and with a consistent effect also on the velocity field. Similarity rules for stratification were developed in [5] analyzing the different behavior of water and sodium in the same facility. This analysis addressed stratification in upper plenum and cannot be easily applied to flows in conduits where higher velocities and wall effects might impact the final considerations.

In this framework, a one-dimensional evaluation was developed [6] to address the influences of the heat transferred from the wall and between the two layers in a fluid flowing in conduits. The evaluation demonstrated that, while the effect of the wall does not greatly affect the evolution of the phenomenon, the introduction of heat transfer between the two layers reduces the persistence of the process and affects its dynamics. Nevertheless, the same author showed that the stratification process is dominated by the hydrodynamic aspect, in particular in the case of low 
Reynolds number conditions; therefore, the performance of water experiments (and water simulations), could provide an important preliminary insight for more detailed experimental and numerical analysis.

Following this example, several experimental mockups were built for the study of stratification behavior in conditions typical in SFR design through the employment of water as a working fluid. In this work, we focused on the results provided by Viollet [7], [8] and [9], whose facility is schematically shown in Fig. 1.

We chose to focus on this experiment for the following reasons:

1) the geometrical similarities with SFR hot leg (e.g. horizontal piping and upward bend);

2) the analogy of the transient dynamics (temperature increase from the top);

3) low flow conditions;

4) high temperature variation in comparison to the inertial forces.

Regarding the last two points, the transient dynamics could be represented by using two non-dimensional numbers, respectively Reynolds $(\mathrm{Re})$ and Froude $(\mathrm{Fr})$ numbers.

It is now clear how the above scheme leads the present evaluation in a zone (transitional Reynolds number and buoyancy driven-flows) which is a priori out of the validity range of the Reynolds average Navier-Stokes (RANS) analysis.

The present work aims first to demonstrate the performance of the usually employed computational fluid dynamics (CFD) models in transitional Reynolds conditions to study the stratification benchmark, which represents the main objective of the present analysis. CFD itself is considered a useful tool to clarify the experimental data through the visualization of the flow field.

\section{A ONE-DIMENSIONAL APPROACH TO STRATIFICATION}

As previously discussed, a one-dimensional model could represent a practical instrument to elucidate the physics of stratification, the parameters controlling the transient, and the role of conductivity during the process.

As shown by Dhir [6], let us consider a horizontal pipe in a gravitational field as shown in Fig. 2. With reference to Fig. 2, when a small volume of fluid at the inlet increases its temperature due to a linear temperature up-ramp (linear transient), the volume will tend to move upward because of density difference creating two layers of different temperature. Through a balance between the potential and kinetic energy between the hot and cold layer, an additional velocity is created in the system acting in the main flow direction for the hotter layer and in the opposite direction for the colder layer $\left(u_{s}\right.$ in Fig. 2).

If the heat transfer $\dot{Q}$ between the two layers is neglected (hydrodynamic model), the velocity $u_{s}$ can be expressed as

$$
{ }^{1} u_{s}=0.5 \sqrt{g \beta \Delta T D},
$$

and from the definition of the $F_{r}$ number

$$
F_{r}=\frac{u_{0}}{\sqrt{g \beta \Delta T D}},
$$

it is clear that in these conditions, for an $F r$ number small enough, the stratification velocity $\left(u_{s}\right)$ exceeds the initial velocity condition; therefore, the cold layer moves backwards.

In the above situation, it is obvious that, from the hydrodynamic point of view, there is essentially no limit to the stratification persistence, because the applied temperature at the inlet is not transferred to the colder layer. This is shown in Fig. 3 with the non-dimensional temperature, where $T_{i}$ is a generic temperature value at

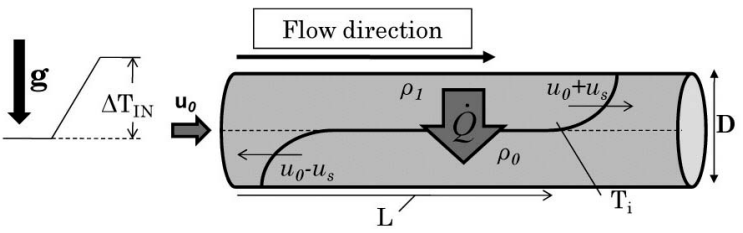

Fig. 2. One-dimensional Model. The Heat Transfer between the Layers $\dot{Q}$, is Neglected in the Hydrodynamic Model.

\footnotetext{
${ }^{1}$ The value of the constant 0.5 , refers to the monodimensional model discussed above. Therefore, in a real experiment (three-dimensional pipe) the value could slightly change [6].
} 
distance $L$ in the hot layer $\left(T_{h}\right)$ or cold layer $\left(T_{c}\right)$. The solid line represents the hot layer temperature, which follows exactly the linear up-ramp with a delay time $t_{0}$ based on the distance $L$. Referring to the present case (low $F_{r}$ number), the temperature in the cold layer does not increase because no heat transfer exists from the inlet or from the hot layer.

However, as already underlined, neglecting the heat transferred between the two layers could represent too large an approximation. Therefore, considering the heat transferred (thermal model), the temperature evaluated at distance $L$ inside the domain, will show a smoother profile compared to the linear transient applied. In Fig. 3 a qualitative evaluation of the non-dimensional temperature history of the hot $\left(T_{h}\right)$ and cold $\left(T_{h}\right)$ layers (faster and slower front respectively) is shown in relation to the time history.

Finally, even though it is only qualitative, this figure shows that after a linear temperature ramp at the inlet, and in particular during transitional $R e$ number conditions, two independent layers might be created; therefore, stratification evaluation in the optic of nuclear reactor safety analysis and in SFRs deserves particular attention.

\subsection{A Detailed Model Validation}

In the further evaluations the CFD code employed will be STAR-CCM+ 5.02.009 [10]. The equations shown in the next chapter will be solved in second-order accuracy in space, and second order advancement in time. The flow is solved as incompressible and the connection between continuity and momentum equations is achieved with Rhie-Chow pressure-velocity coupling combined with the SIMPLE algorithm [10].

\subsection{Mathematical Model}

In the present work the Navier-Stokes equations are solved in their Reynolds average form (where the angle brackets indicate the ensemble average):

$$
\frac{\partial\left\langle U_{j}\right\rangle}{\partial x_{j}}=0
$$

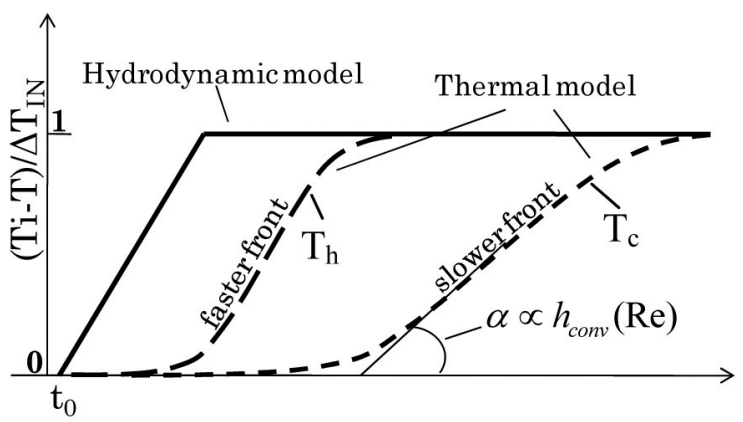

Fig. 3. Qualitative Temperature Time History. Solid Line Represents the Hydrodynamic Model, Dashed Lines Refer to the Thermal Effects (Thermal Model) on Temperature Profile.

$$
\begin{gathered}
\frac{D\left\langle U_{j}\right\rangle}{D t}=-\frac{1}{\rho} \frac{\partial\langle P\rangle}{\partial x_{j}}+\frac{\partial}{\partial x_{i}}\left(v \frac{\partial\left\langle U_{j}\right\rangle}{\partial x_{i}}-\left\langle\overline{u_{i} u_{j}}\right\rangle\right)+g \beta\left(\langle T\rangle-T_{0}\right) \delta_{i 2}, \\
\frac{D\langle T\rangle}{D t}=\frac{\partial}{\partial x_{i}}\left(\frac{\kappa}{c_{p} \rho} \frac{\partial\langle T\rangle}{\partial x_{i}}-\left\langle\overline{\theta u_{i}}\right\rangle\right),
\end{gathered}
$$

where $\left\langle\overline{u_{i} u_{j}}\right\rangle$ and $\left\langle\overline{\theta u_{i}}\right\rangle$ need closure, and $P$ is written as $p+$ $\rho_{0} g\left(x-x_{0}\right)$, where $x_{0}$ represents the reference altitude located at the domain outlet. The body force introduced by gravity was treated with an equation of state. The mentioned equation is obtained by writing the Taylor series for $\rho$ as a function of $T$, considering the pressure constant and truncating at the first order. The fluid is treated with variable density, and the density function is introduced in the code as

$$
{ }^{2} \rho(T)=\bar{\rho}-\bar{\rho} \bar{\beta}(T-\bar{T}) .
$$

When dealing with flow experiencing streamline curvature, it is fundamental to be able to predict the secondary vortices introduced by velocity gradients. The computation of those phenomena through non-linear eddy viscosity models (NLEVM) was demonstrated [11] to achieve a better prediction of the secondary flows and the overall velocity field in comparison with a full resolution of the complete Reynolds stresses (e.g. RSM). Therefore, in the present work, a non-linear cubic relationship for the Reynolds stresses $\tau_{\mathrm{ij}}$ will be employed as shown hereafter:

$$
\begin{aligned}
& \left\langle u_{i} u_{j}^{\prime}\right\rangle=\frac{2}{3} k \delta_{i j}-v_{t} S_{i j}+C_{1} v_{t} \frac{k}{\varepsilon}\left[S_{i k} S_{k j}-\frac{1}{3} \delta_{i j} S_{k l} S_{k l}\right]+C_{2} v_{t} \frac{k}{\varepsilon}\left[\Omega_{i k} S_{k j}-\frac{1}{3} \delta_{i j} \Omega_{j k} S_{k i}\right] \\
& +C_{3} v_{t} \frac{k}{\varepsilon}\left[\Omega_{i k} \Omega_{j k}-\frac{1}{3} \delta_{i j} \Omega_{k l} \Omega_{k l}\right]+C_{4} v_{t} \frac{k^{2}}{\varepsilon^{2}}\left[\Omega_{l j} S_{k i}+\Omega_{l i} S_{k j}\right] S_{k l} \\
& +C_{5} v_{t} \frac{k^{2}}{\varepsilon^{2}}\left[S_{k l} S_{k l}-\Omega_{k l} \Omega_{k l}\right] S_{i j}
\end{aligned}
$$

where $S_{i j}$ is the strain tensor, $\Omega_{i j}$ is the rotation tensor, and $k$ and $\varepsilon$ are scalar variables evaluated through two additional transport equations. The constants in eq. (7) refer to the formulation given in [12].

The closure of the turbulent heat flux is treated through the isotropic eddy diffusivity model:

$$
\left\langle\overline{\theta u_{i}}\right\rangle=-\frac{v_{t}}{\sigma_{T}} \frac{\partial T}{\partial x_{i}},
$$

where $\sigma_{T}$ represents the turbulent Prandtl number, which is assumed to be constant and equal to 0.9 .

The authors of the present work are aware that more accurate modeling of the turbulent heat flux are available which could be more suitable for the present investigation

${ }^{2} \bar{\rho}=997.561\left[\mathrm{~kg} / \mathrm{m}^{3}\right], \bar{T}=300[K], \beta=3.91427 \cdot 10^{-4}[1 / \mathrm{K}]$ 
as shown by [13]. Nevertheless we began the present work focusing on the creation of stratification and its persistence in the horizontal region. This region is demonstrated to be stable; therefore, assuming the turbulent heat flux components aligned with the temperature gradient (refer to eq. (8)), it might represent an acceptable approximation for a preliminary evaluation. In the present model, the effect of gravity is not directly introduced inside the Reynolds stresses definition, but it appears in the transport equations for $k$ and $\varepsilon$ [10].

In relation to the above turbulence modeling, it was previously expressed how this is developed and formally applicable inside the range of fully turbulent regimes. This is true both for the turbulence modeling and for the treatment of the region close to the wall. In this optic therefore, if we pursue the employment of URANS equations in a transitional range, we need to enlighten the modeling performance against benchmarks of simpler problems characterizing the central transient, that is, both an isothermal fully developed straight pipe and sharply bent pipe at transitional $R e$ as described in the next two subsections.

\subsection{Wall Treatment: Fully Developed Pipe at Transitional Re}

When dealing with phenomena at low Reynolds numbers, the physical behavior of fluids shows discrepancies in comparison with the well known fully turbulent flows.

In the case of a low-Reynolds-number flow in a fully developed straight pipe for example, the non-dimensional velocity profile confirms discrepancies from the universal law of the wall, whose equations are shown in Fig. 4. Those results were revealed in the DNS calculations [14] and experimental results [15], indicating that the velocity

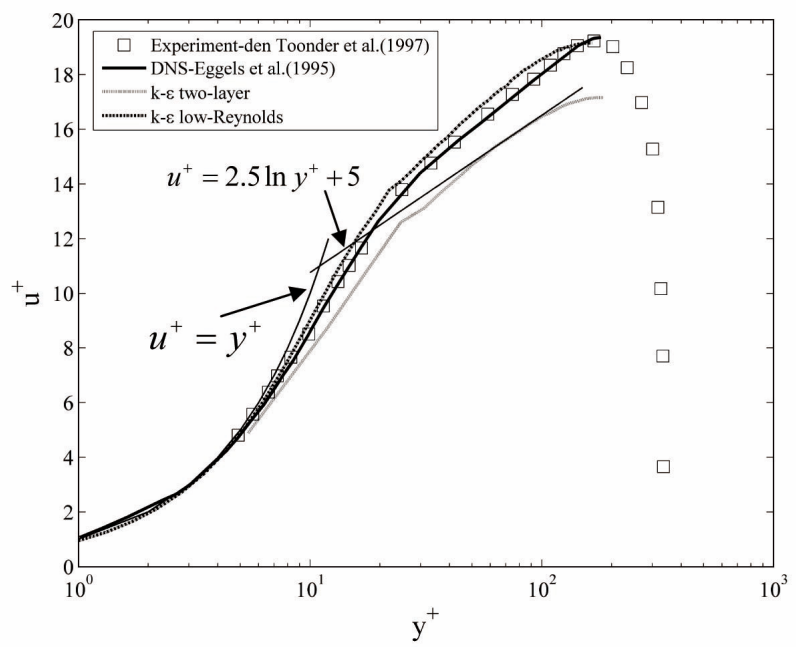

Fig. 4. Non-dimensional Profile at $\operatorname{Re}=5000$. Evaluation of RANS Calculation Performance. distribution has a deviation in the logarithmic region (Fig. 4). Therefore, it is clear that the employment of models (e.g. wall functions, two-layer [16] and low Reynolds formulation [12]) which are based on theoretical and experimental analysis of fully turbulent flows is not obvious to provide satisfying results in wall-bounded conditions.

In further analysis, two basic approaches are employed to solve the region close to the wall, namely two-layer (Wolfshtein [16]) and low-Re as shown by Lien [12]. Simulation was carried out with a three-dimensional pipe of $\operatorname{Re}=5,000$ for water with periodic conditions. Note that the employment of a low-Re model leads the calculation to a bistable condition. Depending on the turbulence initialization, the flow becomes either turbulent or laminar. In Fig. 4, only the turbulent data are shown because it is relevant for the study. The results show good agreement with the DNS results.

On the other hand, the two-layer model formulation predicts a turbulent velocity profile with a shape typical of a fully turbulent regime.

Nevertheless, the flow in a bent pipe is not developed, and its evaluation at transitional Reynolds numbers introduces further uncertainties for the modeling which requires a further investigation in isothermal conditions as validation.

\subsection{Transitional Reynolds Number Flows in Bent Geometries}

Bent pipes are an intrinsic characteristic of nuclearreactor piping-system design in relation to the accommodation of thermal stresses during normal and off-normal operations. Due to design constraints, the piping geometry is likely to be characterized by the presence of very sharp bends.

Fluid flow in a sharp bent pipe is demonstrated [17] to introduce a separation region with flow recirculation, which is found to introduce oscillatory behavior in the streamlines and instabilities of the secondary flows. In general, the sharper the bend is, the larger the recirculation region and the greater the instability. While the employment of URANS for fully turbulent flow in bent pipes is able to reproduce such instability [18], to the best of the authors' knowledge, this has never been demonstrated for transitional Reynolds conditions. Thus, the next validation aims to determine whether the employment of CFD through RANS is able to capture the velocity profile and the introduced oscillations in an isothermal flow during transitional $\mathrm{Re}$ in a sharp bend.

The simulation refers to the data published in [19][20], whose geometry, defined by $R / d=1$, is shown in Fig. 5 . The flow $R e$ number is 4900, and the geometrical domain was built with $3 \mathrm{~d}$ at the inlet and $20 \mathrm{~d}$ at the outlet. Fully developed velocity profiles, as calculated in chapter 2.3, were applied as inlet conditions for both models.

The results shown in Fig. 6 a) and b) demonstrate that RANS modeling is able to capture the characteristic velocity profiles in the radial and streamwise direction in 
the region of recirculation ( $1 \mathrm{~d}$ from the bend outlet).

Fig. 6 a) shows that, while the creation of two peaks of velocity is well predicted, less accuracy is found in the region between the accelerated fluid and the recirculation zone with a general underprediction of the computed results.

It has to be noted, however, that for the low Reynolds formulation on the wall, transient calculation should be employed to achieve an acceptable level of convergence; therefore, the results provided are the time averaged (in Fig. 6 referred to as unsteady results). This shows that

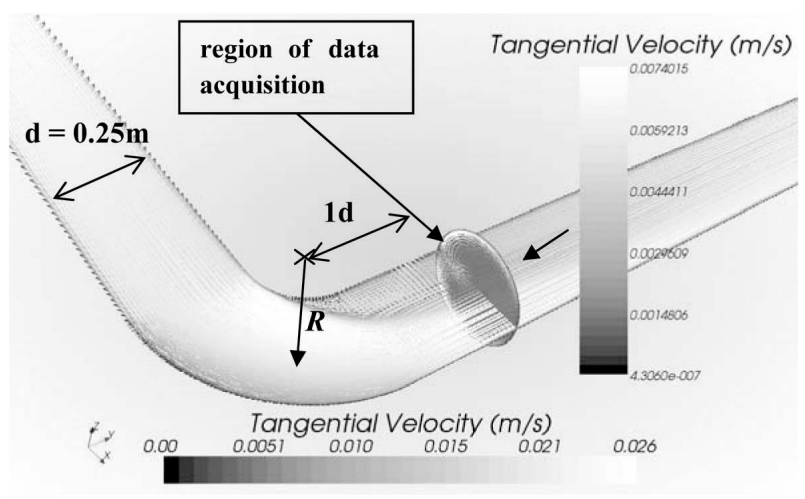

Fig. 5. Geometry and Velocity Vectors. Tangential Velocity Refers to the Vectors on the Cut Section at the Region of Data Acquisition where Secondary flow of the First Kind Could be Observed. this wall treatment approach is potentially able to compute the oscillations which are detected from experiments and large eddy simulation (LES). However, the computed oscillations affect only a small region at the bend outlet, while the experimental data and LES both show a wider influence in the domain. This limitation can be attributed to the present formulation of the damping functions.

The quantitative results confirm these anticipated doubts. The introduction of the bend makes the results of velocity at one diameter from the bend exit tangent similar between the models, and no influence on the inlet condition is found between them. For this reason, in the next section, the $k-\varepsilon$ cubic two-layer model will be employed for the experimental validation.

\section{RESULTS: STRATIFICATION}

The objective of the present work is not to demonstrate the entity of thermal stratification in sodium because detailed experiments for pipes with curvature are currently lacking. The aim of this study is to demonstrate the ability of CFD, through URANS modeling, to capture the main phenomena of stratification in water during a transient and detect the source of uncertainty given by the modeling. The complete validation of the phenomenon is vital to drive model improvement so that future results for sodium flow can be achieved with a higher reliability.

In relation to this goal, the experiment previously introduced [7], whose geometry is shown in Fig. 1, is studied. At time $t=0 \mathrm{~s}$, a linear positive temperature gradient is applied at the inlet for $\Delta t=106 \mathrm{~s}$ (nondimensional time $\Delta \tilde{t}=\Delta t \cdot \bar{V} / D=7.5$ ).

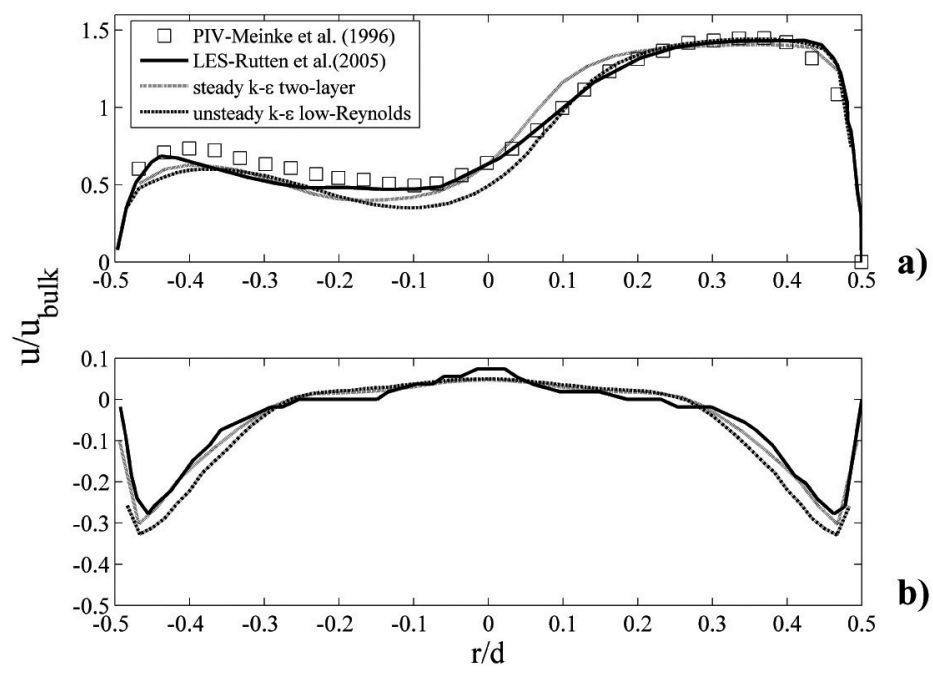

Fig. 6. Velocity Profile Comparisons at $1 \mathrm{~d}$ from the Inlet Tangent. a) shows $v_{x}$ Velocity on the Symmetry Plane, b) shows $v_{y}$ on the Line Normal to the Symmetry Plane Passing Through the Centerline. 
The non-dimensional numbers which govern the transient are $R e=5000$ and $F r=0.25$.

For the geometric discretization, a polyhedral grid has been used with a prismatic layer near the wall boundary as shown in Fig. 7 c). The computational domain is composed of almost 2,500,000 cells which represent the coarse mesh. A finer domain was created and simulated for what concerns the steady-state isothermal conditions of the flow. Since no appreciable difference was found in the steady-state flow, a coarser mesh was employed for the rest of the calculations.

\subsection{Horizontal Region}

In the simulation, the process of stratification, determined by a low $F r$ number, shows the hot fluid moving from the top and gradually deposing on the cold layer. This progression shows a high stability (i.e. absence of Kelvin-Helmholtz instability (KHI)). This behavior can be related to the relatively high temperature difference, resulting in a high buoyancy force (referring to eq. (2)) in comparison with the inertial ones. As shown from dynamic instability analysis for 2D domain fluid of different densities, instability is created for $F r>2$; therefore, from a qualitative analysis, the simulation is in agreement with theory and experience. Fig. 8 a) and b) shows the particular of the horizontal region at time $t=212 \mathrm{~s}$ (equivalent nondimensional time $\tilde{t}=15$ ), where a stable stratification is created. As previously discussed, this region presents a sharp temperature gradient which divides the flow into two layers of almost constant temperature because of the low thermal conductivity of water, which is also reflected in a clearly defined division of velocity between the two layers. The particular of Fig. 8 b) shows that, at this moment, the velocity in the lower region is directed in the $-x$ direction, which means that, as anticipated, (onedimensional modeling) the two layers are completely independent. This is of great relevance because, together with the lack of KHI, it will make the stratification persist for a long time during the transient. Therefore, the great persistence of stratification is created by the following factors:

- Low heat transfer of the water

- No mechanical entrainment in the dividing layer

- Velocity inversion in the cold layer
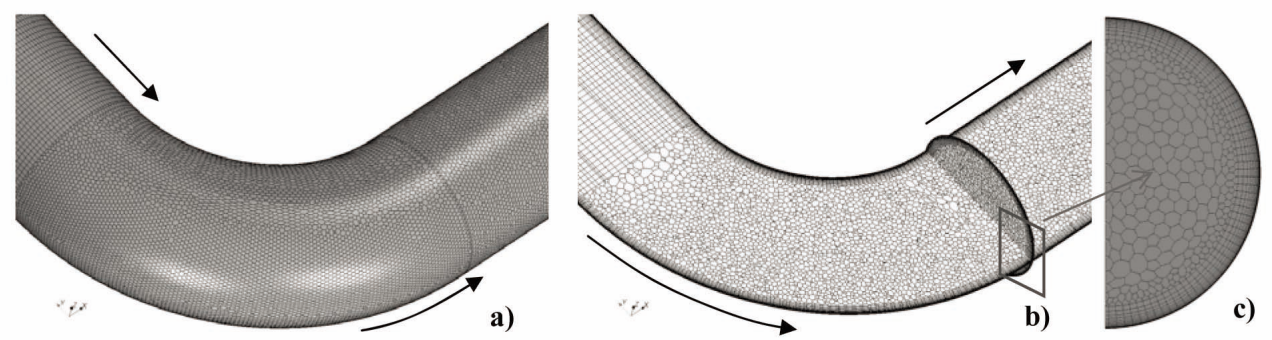

Fig. 7. Mesh Adopted for the Geometry Shown in Fig. 1. The First Bend from the Inlet is Shown in the Above Pictures. The Black Arrows Represent the Flow Direction.
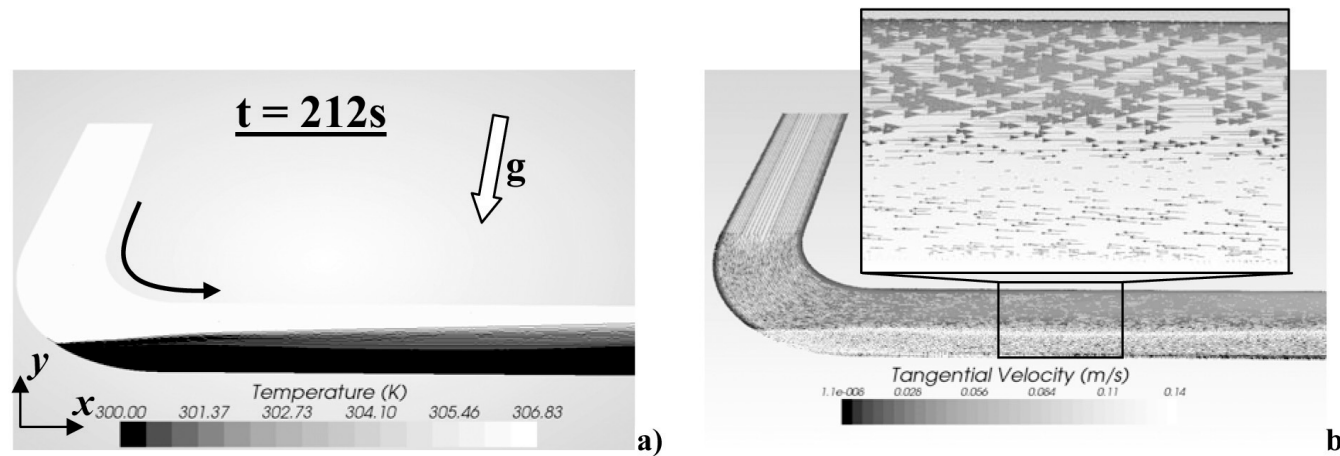

Fig. 8. Temperature a) and Velocity b) Fields at $t=212 \mathrm{~s}$. In b) Blow Up of the Stratified Region Showing the Reverse Flow Creation. In a) the Black Arrow Indicates the Main Flow Direction. 
The temperature and velocity field at time $t=318 \mathrm{~s}$ (nondimensional time $\tilde{t}=22.5$ ) are not shown in this paper because the two fields are essentially the same as the one discussed in Fig. 8. The only difference is introduced by the presence of the bend which redirects part of the flow against gravity.

In these conditions, therefore, we can claim that the persistence of stratification in similar transients is threatened only by the heat transferred between the two layers with the possibility that during such transients this phenomenon in the piping system could assume a high importance and influence.

The results of the experiment show that in the horizontal region from points 3 to 8 the non-dimensional temperature refers to the lowest and highest values, and this indicates the presence of thermal stratification. Calculations demonstrate the ability to reproduce stratification and its persistence. From $t=212 \mathrm{~s}$ to $t=318 \mathrm{~s}$, the non-dimensional temperature is not minimally changed, in complete agreement with the experimental data.

This achievement represents an enhancement of the computational results in comparison with the $2 \mathrm{D}$ numerical simulation which was performed by the author of the experiment as a validation [7].

\subsection{Vertical Region}

Note that, in SFRs, after a change in the elevation to enhance the natural circulation, a long horizontal pipe section leads to the intermediate heat exchanger. Therefore, despite the fact that the main objective of the work is to address the flow in the horizontal region, it is necessary to particularly consider the vertical part since it can affect the possible creation of stratification in the zone subsequent to the change in elevation. The evaluation of the temperature distribution in the vertical region, therefore, provides important information on the possibility of stratification creation in a hypothetical horizontal zone which could affect the whole transient in a nuclear power plant.

From Fig. 9 b) and c), it is clear that the prediction in the vertical part of the pipe in both instants of time shows high discrepancies from the experimental data. The experimental data show that the temperature between the hot and cold walls becomes uniform.

Calculations shown in Fig. 9 b) and c) show that the present evaluation cannot reproduce the local values of the temperature on the hot and cold wall even though the general trend is reproduced. Nevertheless, simulations provide further information in comparison to the experimental data. Note that an inversion of temperature between the inner and outer wall appears. This inversion seems to result from the oscillations of the temperature current. Even though comparisons with this finding are not available, similar considerations of sharp bends in isothermal flows suggest that instabilities are introduced and subsequently incremented by the buoyancy term. Fig. 10 demonstrates how the flow in this region shows oscillatory behavior in the $x-y$ plane, which persists in time and which is responsible for the temperature inversion.

Moreover, it should be noted that, in the vertical region, the flow behavior becomes completely threedimensional; therefore, the only evaluation of the symmetry plane might mask some important phenomena of the flow. In Fig. 11 and Fig. 12 the temperature field and velocity vectors at two elevations in the vertical pipe (points 9-10 and 11-12) are shown. These figures show that the presence of a bend in the direction opposite to

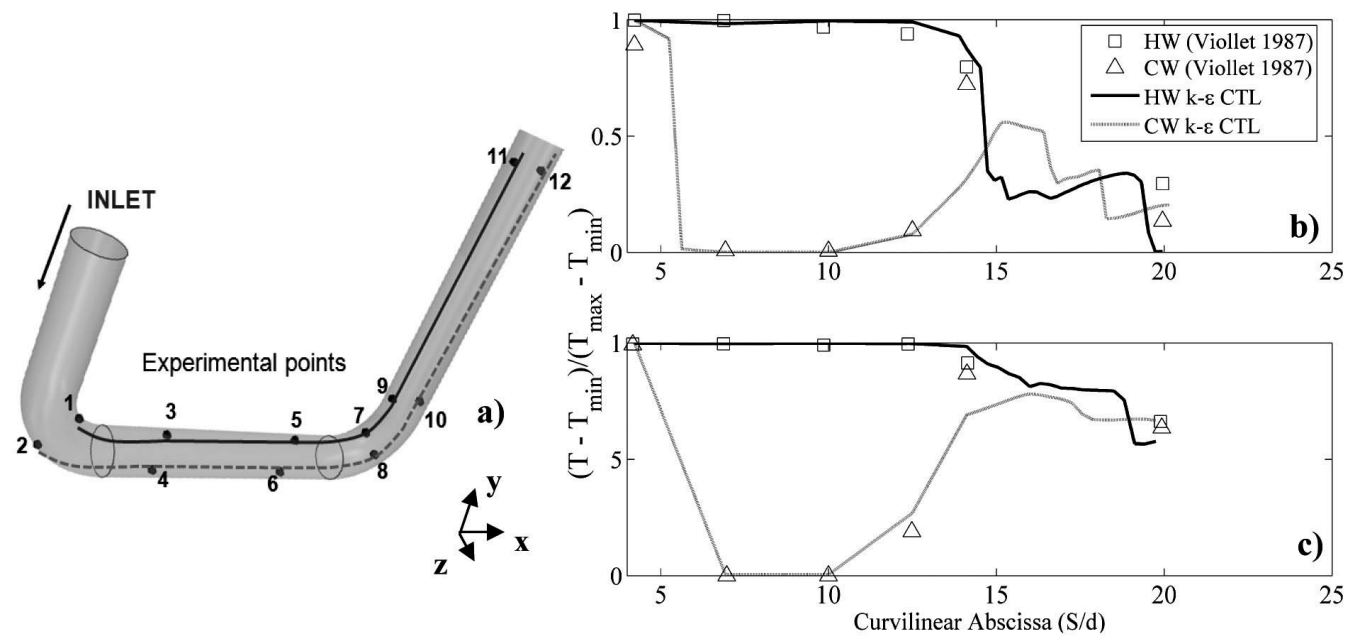

Fig. 9. Temperature Profile on the Hot and Cold wall. a) Temperature Monitoring Points. Comparison of the Results at $t=212 \mathrm{~s}$ in b), and $t=318 \mathrm{~s}$ in $\mathrm{c}$ ). 

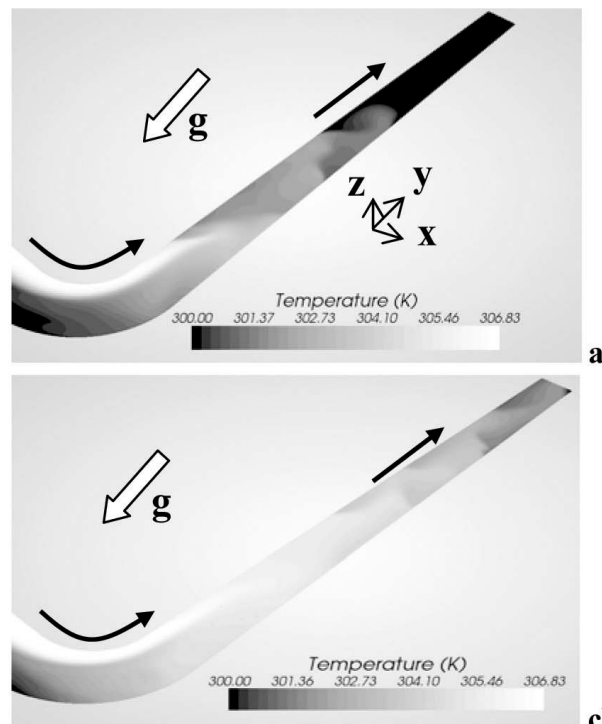

c)

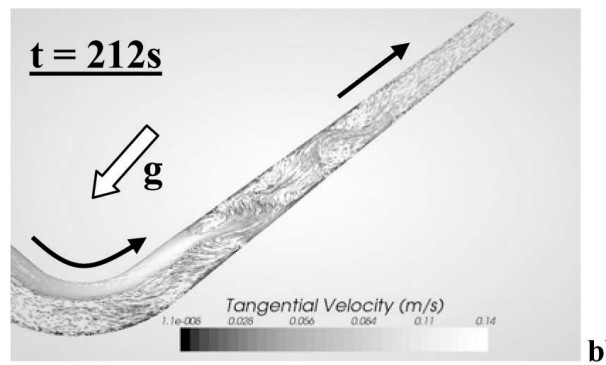

b)

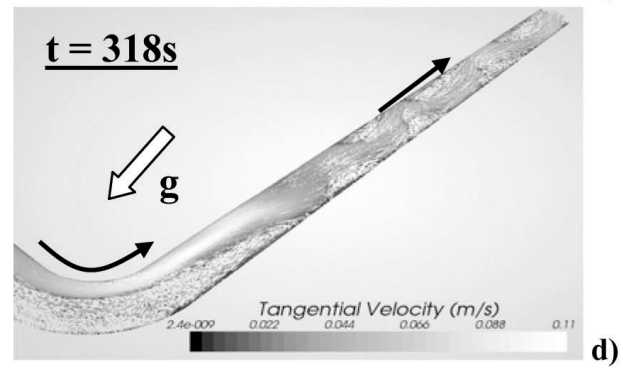

Fig. 10. Temperature Contours a); c) and Velocity Vectors b); d) on the Symmetry Plane at Two Different Instant in Time for the Instability Visualization of the Vertical Region. a) and b) refer to $t=212 \mathrm{~s}$; ) and $d$ ) $t=318$ s. Closed Arrows Indicate the Flow Direction and Open Arrows Gravity Direction.

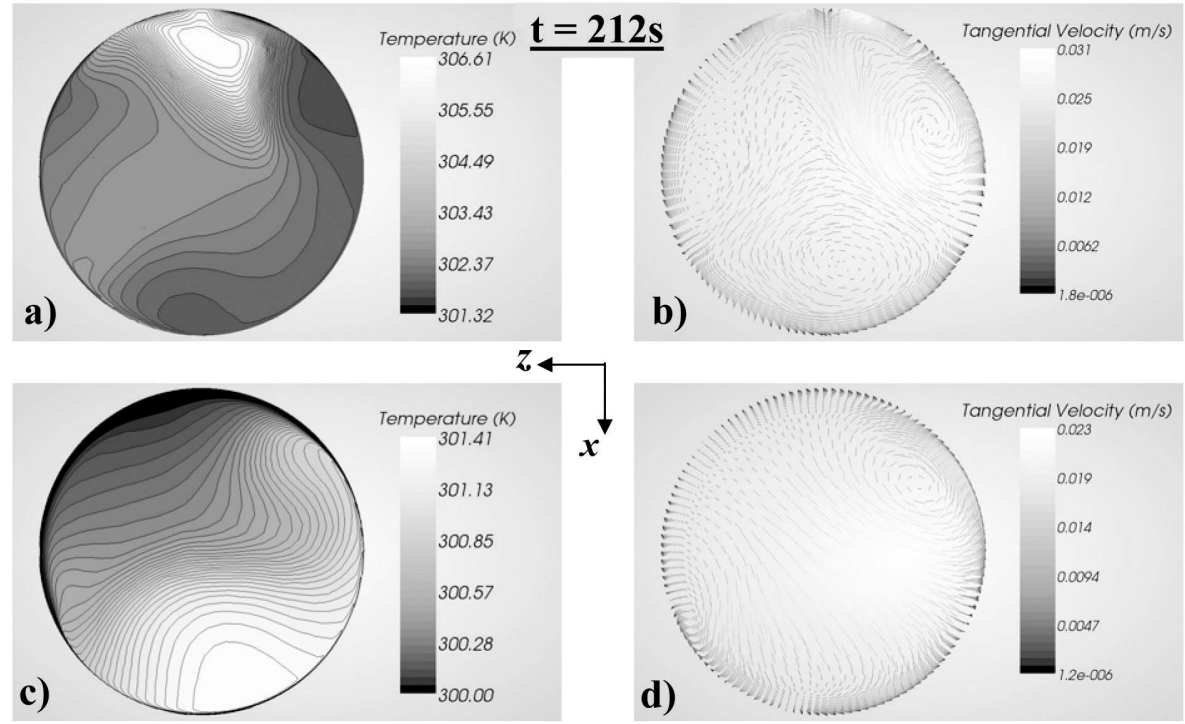

Fig. 11. Temperature Field and Velocity Vectors at Time $t=212$ s. a) and b) Refer to the Plane Passing Through the Points 9 and 10 (Fig. 9 a)); c) and d) Through 11 and 12.

gravity introduces an asymmetry of the secondary flows and the related temperature field. The temperature contours (Fig. 11 a) and c)) show the changes in the circumferential location of the density current, clarifying that instability and oscillatory motion experiences a component in the $x$-z plane also. This phenomenon is magnified in Fig. 12 a) and c).

Based on these considerations, another important 


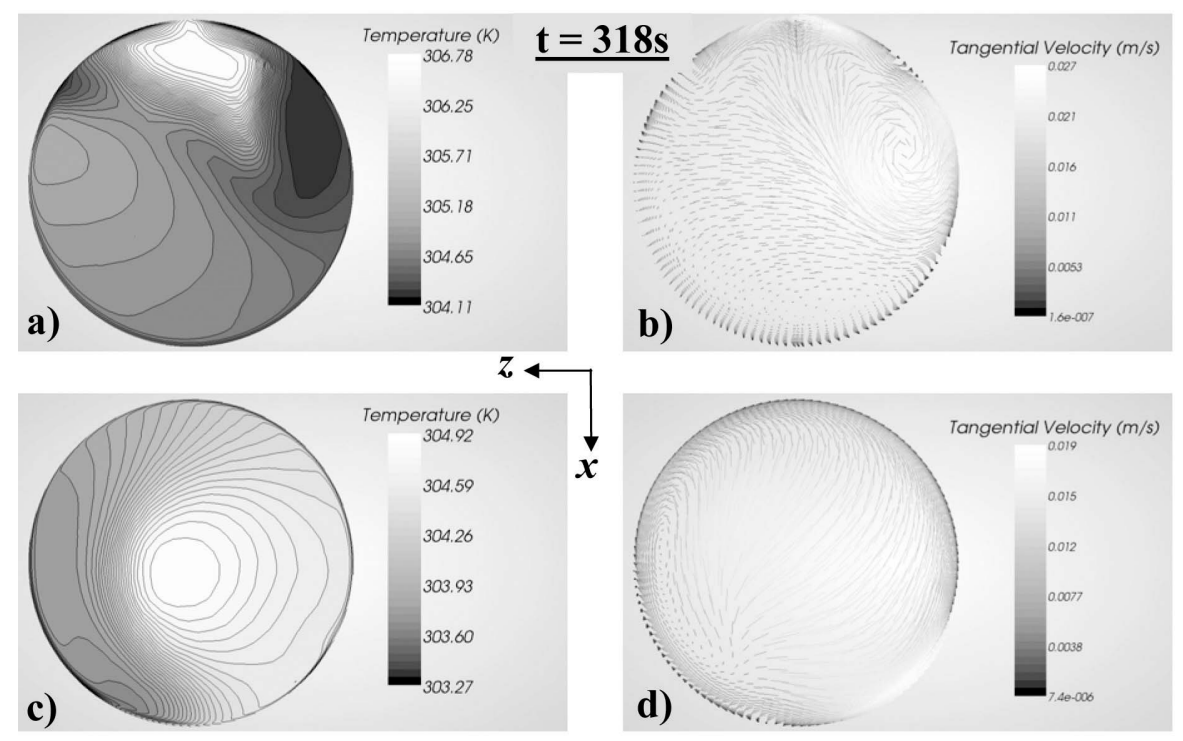

Fig. 12. Temperature Field and Velocity Vectors at Time $t=318 \mathrm{~s}$. a) and b) Refer to the Plane Passing Through Points 9 and 10 ; c) and d) Through 11 and 12 .

conclusion can be drawn. In relation to Fig. 9, one of the main conclusions of the author of the experiment was that the temperature field reached an almost uniform temperature distribution in the region at the outlet bend tangent. Our results show instead that the introduced oscillations of the flow in the $x-z$ plane can lead to a temperature difference in the direction normal to the symmetry plane. Therefore, it appears obvious that the sole consideration of the temperature distribution on the symmetry plane can fake some important aspects of the phenomena appearing in this region.

From the present evaluation, it seems that the density currents survive longer in the domain and longer in time. This is vital in the stratification evaluation for both water and sodium.

Finally, it was already mentioned that an improved turbulent heat flux model could be thought to be more reliable for the present analysis. Even though this improvement is likely to enhance the agreement of the results with the experimental data, this is not thought to substantially affect the flow behavior; rather, it influences the local value of the temperature. In [13], for example, the employment of a more sophisticated turbulent heat flux model results in a better prediction of the Nusselt number, while the overall prediction of the velocity and temperature field is not strongly affected. These conclusions appear to satisfactorily demonstrate that the presence of vertical pipe does not make the temperature in the $x-z$ plane completely uniform; therefore, the occurrence of thermal stratification is likely to exist further in the piping system.

\section{CONCLUSIONS}

Simulation of the flow in a U-bent pipe and related geometries has been carried out using the unsteady Reynolds average Navier Stokes approach.

Because of the characteristics of the flow conditions, the present benchmark required validation of the URANS performance in transitional Reynolds number flows. This study aimed to further our understanding of various phenomena created by the analyzed flow regime and geometry, and has presented a detailed CFD analysis in these circumstances. Even though transitional Re analysis might not be essential in many industrial fields, it is extremely important in the nuclear environment, in particular in relation to safety analysis of SFRs during decay heat removal.

Predictions of stratification creation and persistence in the horizontal region of the hot and cold wall were found to be in good agreement with the experimental values. The creation of a recirculation region (stagnant flow) in the horizontal pipe is a leading factor in the stratification phenomenon influencing its persistence.

Moreover, the presence of an upward bend introduces complicated phenomena, in which buoyancy plays an important role in the onset of instability. Despite the differences between the local temperatures in the vertical direction in comparison with the experimental data, analysis shows some novel results regarding density currents. The longer persistence of the density current and related temperature difference extend the possibility of thermal 
stratification for the whole piping system in a reactor.

Future works should be considered for investigation of different damping functions on the wall and employment of a more general turbulent heat flux definition [13]. The more detailed modeling of the flow would finally lead to a more reliable simulation of similar conditions and geometry with a fluid respecting the properties of sodium (i.e. relatively high heat conductivity).

\section{REFERENCES}

[1] NRC, Unexpected piping movement attributed to thermal stratification, Inform. Notice 88-80, October 7, (1988).

[2 ] L. Wolf, Hafner, M. Geiss, E. Hanjosten, G. Katzenmeier, "Results of HDR-experiments for pipe loads under thermally stratified flow conditions," Nucl. Eng. and Des. 137, 387-404 (1992).

[3] A. Talja, E. Hanjosten, "Results of thermal stratification tests in a horizontal pipe line at the HDR-facility," Nucl. Eng. Des. 118, 29-41 (1990).

[4] A. B. Cortesi, G. Yadigaroglu, S. Banerjee, "Numerical investigation of the formation of the three-dimensional structures in stably stratified mixing layers", Physics of Fluids, Vol. 10, No. 6, 1998, pp. 1449-1473.

[ 5 ] M. Ohtsuka, T. Ikeda M. Yamakawa, Y. Shibata, S. Moriya, S. Ushijima, K. Fujimoto, (1988) "Similarity rules of thermal stratification phenomena for water and sodium", $4^{\text {th }}$ International conference on liquid metal engineering and technology, Vol. 2 Sec. 420.1.

[6] V. K. Dhir, R. C. Amar, J. C. Mills, A one dimensional model for the prediction of stratification in horizontal pipes subjected to fluid temperature transient at inlet, Part I \& II, Nucl. Eng. Des. 107, 307-314 (1988).

[ 7 ] Viollet P.L., (1987a) "Observation and numerical modelling of density currents resulting from thermal transients in a non rectilinear pipe", Journal of Hydraulic Research, 25, No. 2 (1987).

[ 8 ] Viollet P.L., (1987b) "The modeling of turbulent recirculating flows for the purpose of reactor thermal-hydraulic analysis", Nucl. Eng. and Des., 99, 365-377 (1987).

[9] Viollet P.L, J.P. Benque, J. Goussebaile, (1987) “Twodimensional numerical modeling of nonisothermal flows for unsteady thermal-hydraulic analysis", Nucl. Sci. Eng.,
84, 350-372 (1987).

[10] STAR-CCM+ Version 5.02 User guide.

[11] E. Baglietto and H. Ninokata, "Anisotropic eddy viscosity modeling for application in industrial engineering internal flows," Int. J. Transport Phenomena, 8, (2006).

[12] F.S. Lien, W.L. Chen, M.A. Leschziner, (1996), "LowReynolds number eddy-viscosity modelling based on nonlinear stress-strain/vorticity relations," Proc. 3rd Symp. on Engineering Turbulence Modelling and Measurements, 27-29 May, Crete, Greece.

[13] S. Kenjeres, K. Hanjalic, "Convective rolls and heat transfer in finite-length Rayleigh-Benard convection: A twodimensional numerical study," Physical Review E, , 79877998, (2000).

[14] J. G. M. Eggels, F. Unger, M. H. Weiss, J. Westerweel, R. J. Adrian, R. Friedrich, F. T. M. Nieuwstadt, "Fully developed turbulent pipe flow: a comparison between direct numerical simulation and experiment," J. Fluid Mech. 268, 175-209 (1994).

[15] J.M.J den Toonder, F.T.M Nieuwstadt, "Reynolds number effects in a turbulent pipe flow for low to moderate Re" Phys. Fluids, 9 (11), November (1997).

[16] Wolfshtein M., "The velocity and temperature distribution in one-dimensional flow with turbulence augmentation and pressure gradient,” Int. J. Heat Mass Transfer, 12, 301-318, (1968).

[17] M.J. Tunstall, J.K. Harvey, "On the effect of a sharp bend in a fully developed turbulent pipe-flow," J. Fluid Mech., 34, part 3, pp. 595-608.

[18] M. Tanaka, H. Ohshima, H. Yamano, K. Aizawa, T. Fujisaki, (2009), "Application of U-RANS to elbow pipe flow with small curvature radius under high Reynolds number condition," Proceedings of International Congress of Advances in Nuclear Power Plants ICAPP 2009, 10-14 May, Tokyo, Japan.

[19] F. Rutten, W. Schroder, M. Meinke, "Large eddy simulation of low frequency oscillations of the Dean vortices in turbulent pipe bend flows," Phys. Fluids, 17, 035107-035107-11 (2005).

[20] Ch. Brucker, "A time-recording DPIC-study of the swirl switching effect on a $90^{\circ}$ bend flow," Proceedings of the Eight International Symposium on Flow Visualization, Sorrento,Italy, 1998. 